ARTIGO DE ATUALIZAÇÃO

\title{
ASSOCIACCÃO DE TERAPIAS E TECNOLOGIAS PARA A GAGUEIRA: AVANÇOS, CONTROVÉRSIAS E DESAFIOS
}

\section{ASSOCIATION OF THERAPIES AND TECHNOLOGIES FOR STUTTERING: ADVANCES, CONTROVERSIES AND CHALLENGES}

\section{Resumo}

No presente trabalho, discutimos como o uso associado de tecnologias, como a realidade virtual, com abordagens de tratamentos modernas, como a terapia cognitivocomportamental (TCC) associada ao mindfulness, pode contribuir para resultados mais eficazes no tratamento da gagueira. Os antigos tratamentos focados unicamente em terapia comportamental, com uso prioritário da terapia de exposição, foram sendo substituídos e/ ou associados a novas abordagens, utilizando a TCC como base do processo de tratamento. Atualmente, protocolos que visam difundir o uso de tecnologias de realidade virtual, videogames e estimulação transcraniana com esse novo foco terapêutico expandido, tendo por objetivo a diminuição da ansiedade antecipatória/ social, juntamente com o ganho de atenção plena e de percepção de gatilhos internos e externos de ansiedade, estão mudando de maneira bastante consistente o panorama do tratamento da gagueira. Neste artigo, vamos considerar diversos trabalhos seminais e novos que demonstram que tecnologias de simulação de situações de vida real são as práticas que têm obtido os melhores resultados no tratamento da gagueira.

Palavras-chave: Gagueira, tratamentos, tecnologia.

\section{Abstract}

In this paper we discuss how the use of technologies (such as virtual reality) associated with modern treatment approaches (such as cognitive behavioral therapy [CBT] with mindfulness) can contribute to more effective results in the treatment of stuttering. Old treatments, which focused solely on behavioral therapy, with priority use of exposure therapy, have been replaced and/or associated with new approaches using CBT as the basis of the treatment process. Currently, protocols that aim to spread the use of virtual reality technologies, video games and transcranial stimulation with this new expanded therapeutic focus, aiming to decrease anticipatory/social anxiety together while at the same time gain full attention and perception of internal and external anxiety triggers, are consistently changing the scenario of stuttering treatment. In this article, we will consider several seminal and new works that demonstrate that simulation technologies of real life situations are the practices that have shown the best results in the treatment of stuttering.

Keywords: Stuttering, treatment, technology.

\section{INTRODUÇÃO}

É inegável que as intervenções cognitivas baseadas em jogos de videogame para o tratamento terapêutico da gagueira são ainda incipientes. Tratamos desse assunto em um trabalho anterior ${ }^{1}$, em que discutimos o estudo de Lavender \& Gromala², que construíram um videogame com o propósito de incrementar a fluidez verbal, utilizando como aporte as abordagens da terapia cognitivo-comportamental (TCC).

No trabalho em questão², os autores avaliaram o uso de videogames para o tratamento da gagueira, utilizando um jogo protótipo chamado Audition, The 
Facultad de Psicología, Universidad Nacional de Córdoba, Córdoba, Argentina. ${ }^{2}$ Consultório particular.

Game (algo como uma audição para uma peça de teatro). Nesse protótipo, a dinâmica de jogo consistia em controlar um personagem que deveria parar em uma audição ou abandonar o seu sonho de tornar-se um ator. O personagem, que é controlado pelo jogador através do mouse, antes de chegar à dita audição, deve resolver uma série de objetivos, passando por uma enorme quantidade de obstáculos durante o seu percurso, com a finalidade de aumentar o seu estresse e a sua ansiedade (por exemplo, andar em um skate, enfrentando o tráfego de automóveis, para chegar à audição). Depois dessa fase inicial, o jogador finalmente é apresentado a uma sala, na qual deve ler em voz alta, durante 30 segundos, um trava-línguas, preservando o ritmo e a harmonia. Se esse desafio é completado com êxito, um novo ciclo de leitura é iniciado, aumentando a dificuldade e ajustando o tempo de exposição, até alcançar o nível máximo de atuação e, assim, ser nomeado como ator dentro do jogo. Depois de 10 minutos de experiência de jogo, os jogadores foram reavaliados através de diversos questionários a respeito da dificuldade do jogo, o prazer de jogar e a ansiedade causada durante o processo. 0 estudo piloto demonstrou que esse jogo em particular era agradável, atrativo e desafiante, como também apresentou uma possível relação entre a antecipação da fala e o estresse. Uma das medidas avaliadas foi a resposta galvânica da pele, que durante os desafios vocais aumentava substancialmente, sugerindo, assim, que a antecipação do desafio vocal pode, na realidade, causar maior estresse do que o desafio real ${ }^{2}$.

Dessa maneira, o presente trabalho tem por objetivo apontar a pertinência das intervenções baseadas em videogames, analisando o contato da gagueira.

Com o intuito de desenhar intervenções efetivas para o transtorno, é fundamental realizar primeiramente uma aproximação conceitual. A gagueira, também conhecida como disfemia ou disfluência, é um transtorno da fluidez, caracterizado pela aparição de paradas repentinas da fala, prolongações, distorções, adição de interlocuções, repetições de sílabas ou circunlocuções verbais, que se produzem ao substituir palavras complexas por outras mais simplificadas ${ }^{3,4}$. Esses sintomas geralmente estão acompanhados de diversos componentes fisiológicos e psicológicos, que se associam a reações involuntárias, como a contração dos músculos faciais, a transpiração corporal, as palpitações, o rubor e o desvio do olhar, entre outros 5 .

A prevalência da gagueira afeta cerca de 3 milhões de pessoas nos EUA ${ }^{6}$, e o seu aparecimento acontece relativamente cedo no desenvolvimento ontogenético: entre os 2 e os 5 anos de idade?. Pelo panorama das contribuições provenientes da epidemiologia, a primeira aparição da gagueira se dá por volta dos 3 ou 4 anos de idade $^{8}$. As dificuldades de associação podem se prolongar até a adolescência e inclusive continuarem de forma pervasiva pela vida adulta, convertendose, por consequência, em prejuízos crônicos?. É importante ressaltar que os dados indicam que esse transtorno é mais frequente em homens ${ }^{10}$ do que em mulheres. A gagueira cobre um amplo espectro de comportamentos estereotipados, tanto ansiogênicos quanto patológicos. Portanto, esse quadro complexo da gagueira associa diversas manifestações cognitivas e comportamentais que são próprias de um transtorno emocional, como a fobia ou a ansiedade social (ver Craig \& $\operatorname{Tran}^{11}$ para uma metanálise do tema). A nossa percepção é de que a dimensão social da gagueira não é menor, e por isso é necessário um enfoque que lide com componentes físicos (do aparelho fonador), emocionais (relacionados à ansiedade), comportamentais (expressão da fala) e cognitivos (da organização do discurso).

O tratamento tradicional da gagueira se apoia em dois principais eixos, fonoaudiologia ou terapia da expressão da fala e TCC ${ }^{12}$. À luz dos recentes avanços (para uma revisão exaustiva sobre tratamentos, ver Brignell et al. ${ }^{13}$ ) do uso de videogames para a saúde, é pertinente repensar o uso desses dispositivos para avaliação e tratamento de diversas variáveis clínicas. Nesse sentido, circulam há alguns anos diversos dispositivos que geram sons ou ruídos enquanto o indivíduo fala. O objetivo dessa abordagem é desconectar o ato de falar do ato de escutar, evitando que o feedback auditivo da expressão da fala, de alguma forma, possa gerar ansiedade por conta de pequenas falhas e, consequentemente, aumentar a ansiedade social, prejudicando ainda mais a fluência do paciente com gagueira. Entretanto, a eficácia desses dispositivos para uma abordagem específica para a gagueira ainda é limitadá. 
Abordagens recentes sugerem que a TCC, complementada com técnicas de atenção plena, como o mindfulness, poderia ser eficaz para o tratamento da disfluência que acomete o paciente com gagueira. Nesse sentido, Mongia et al. ${ }^{14}$ sugerem que o mindfulness, além de ajudar os pacientes disfluentes a ganhar consciência e identificar o que fazem enquanto gaguejam, pode auxiliar de maneira simultânea a gerar consciência das respostas dos ouvintes. O mindfulness intervém em uma melhor gestão do controle atencional e concomitantemente induz a mudanças significativas na percepção dos pensamentos disfuncionais associados ${ }^{15}$.

No âmbito dessa técnica, também foi relatado que a exposição imaginativa influencia na aceitação dos pensamentos intrusivos ansiosos, possibilitando, desse modo, uma regulação emocional mais eficiente ${ }^{16}$. Em seu trabalho, Rodríguez-Carvajal et al. ${ }^{16}$ fizeram um acompanhamento de um paciente e evidenciaram melhoras significativas no aumento da sua fluidez da fala através do uso da exposição imaginativa, que foi essencial para evitar as recaídas e manter os ganhos efetivos da intervenção em outros contextos.

Geralmente, o protocolo de intervenção combinando TCC com mindfulness, dependendo da gravidade dos sintomas, pode oscilar entre 3 meses e 3 anos de tratamento, com variação do número de sessões. Adicionalmente ao tratamento, sessões de acompanhamento são necessárias para evitar recaídas. Associadas, essa abordagem de TCC e abordagens baseadas em mindfulness poderiam ser estratégias factíveis para o tratamento.

Por exemplo, está bem estabelecido que as terapias da terceira onda, como a terapia de aceitação e compromisso (ACT), induzem uma maior flexibilidade psicológica em diversos transtornos, ampliando esses benefícios à disfluência, como sugere uma série de estudos sobre o tema (Harley ${ }^{17}$ oferece uma revisão detalhada sobre mindfulness e gagueira). De forma similar, Beilby et al. ${ }^{18}$ aplicaram um programa de treinamento em mindfulness em adultos que gaguejavam. Os resultados mostraram que os efeitos da intervenção melhoraram diversos aspectos vinculados com o gerenciamento do corpo, das emoções, dos pensamentos e em relacionamentos interpessoais em situações sociais, com resultados consistentes depois de 3 meses do término da intervenção. De forma complementar, De Veer et al. ${ }^{19}$ avaliaram os efeitos de implementar um programa de treinamento de mindfulness para a redução do estresse em 37 pessoas com gagueira. Os resultados desse estudo destacaram uma diminuição nos níveis de estresse e ansiedade durante eventos de exposição social, aumentando a capacidade dos participantes de gerenciar eficientemente o locus de controle interno.

A robustez dessa técnica, assim como a facilidade da sua implementação, tanto em um nível individual como grupal, possibilitam importantes implicações na qualidade de vida do paciente, complementando enfoques tradicionalmente instaurados (TCC, terapia da fala/fonoaudiologia, dispositivos de realidade virtual, entre outros).

Recentemente, e considerando a heterogeneidade das abordagens vigentes, surgiram dispositivos de biofeedback que demonstraram resultados promissores para o tratamento de pessoas com disfluência. Especificamente, Modifying Phonation Intervals 2 (MPI$2)^{20}$ é uma abordagem inovativa de biorretroalimentação. A terapia baseada na evidência permite que as pessoas que gaguejam possam utilizar essa biorretroalimentação adaptada para modificar a forma como utilizam suas pregas vocais para reduzir a própria gagueira. Esse novo padrão de fala acaba promovendo uma maior fluidez e aumenta a confiança em situações de fala exigentes. Os participantes mostraram uma maior autoidentificação como falantes normais (isto é, uma menor autoidentificação como gagos), uma redução dos intervalos de fonação curtos e um aumento do uso de intervalos de fonação de maior duração.

Da mesma forma, é pertinente realizar uma menção especial a uma recente abordagem que poderia facilitar a abertura de novas linhas de investigação em torno do tratamento dessa patologia da fala. É o caso da estimulação transcraniana por corrente direta (ETCD) - do inglês transcranial direct-current stimulation (tDCS) - como coadjuvante da terapia da fala. A abordagem consiste em utilizar uma corrente elétrica fraca, aplicada às regiões do cérebro associadas à gagueira, através de eletrodos, que modulam o potencial de membrana em repouso dos neurônios no córtex. Foi levantada a hipótese de que, ao aplicar o ETCD enquanto o paciente estivesse falando fluentemente (durante um processo 
Facultad de Psicología, Universidad Nacional de Córdoba, Córdoba, Argentina. ${ }^{2}$ Consultório particular.

de TCC), os circuitos cerebrais poderiam ser ativados para facilitar a fluência na fala e, consequentemente, um aumento da fluência poderia ser melhor mantido em adultos que gaguejam² ${ }^{21}$.

Em relação às intervenções baseadas em videogames, Stamurai é um videogame de livre acesso (https:// stamurai.com/) que permite que as pessoas com disfluência treinem a sua fala com uma série de exercícios, como prática de leituras (ler em voz alta o conteúdo, gravar a voz e reproduzi-la em sequência), assim como para as vogais e as consoantes (praticar os sons em lugar das palavras) e fluidez da fala (identificação consciente do fluxo da fala, respondendo a perguntas).

Diversos grupos de investigação têm contribuído para a construção de metodologias e diretrizes para a criação de jogos terapêuticos e/ou intervenções comportamentais com objetivos de tratamento em áreas da saúde e da clínica psicológica (ver, por exemplo, Rivero ${ }^{22}$. Por exemplo, Baranowski et al. ${ }^{23}$ apresentam uma série de critérios técnicos que se podem utilizar para construir um jogo que crie generalização de aprendizados terapêuticos do jogo para a vida real. Nosso próprio grupo desenvolveu um método para a criação de videogames terapêuticos com enfoque na reabilitação neuropsicológica do $\mathrm{TDAH}^{24}$.

No âmbito dos videogames usados com finalidades terapêuticas, estes podem ser classificados em dois grandes grupos: os jogos de treino repetitivo de habilidades específicas (isto é, falar vogais ou consoantes); e os jogos que têm a intenção de se aproximar o máximo possível a simulações de situações cotidianas reais, como o jogo de Lavender \& Gromala² (para uma discussão sobre esse tema, ver Rivero et al. ${ }^{24}$ ). Fundamentalmente, esses videogames que usam simulações de vida cotidiana se utilizam de intervenções respaldadas empiricamente pelas terapias de exposição, que consistem no controle e na repetição de estímulos aversivos para o paciente (isto é, falar em público, em voz alta) ${ }^{25}$.

Para além das terapias comportamentais clássicas, como a terapia de exposição, é essencial avançar em direção às TCC associadas a videogames. Klinger etal. ${ }^{26}$ buscam se aproximar dessa necessidade metodológica. Eles compararam a eficácia clínica de um dispositivo de realidade virtual com o da TCC (enfoque terapêutico tradicional). Os resultados indicaram não haver diferenças de efeito significativas entre a TCC tradicional e a baseada em realidade virtual. Parecenos que o próximo passo natural é a associação de TCC e mindfulness com jogos de simulação de situações cotidianas e de vida real de pacientes com gagueira. Esse passo, por mais natural que seja, envolve uma série de dificuldades relacionadas a barreiras e restrições metodológicas da própria pesquisa científica, econômicas, estatísticas e de engajamento terapêutico ${ }^{24}$.

Por fim, a exposição repetida e sistemática a diversos cenários sociais em um dispositivo de realidade virtual é uma aposta importante para o futuro do tratamento da gagueira, já que aumenta a sensação de segurança e mitiga a típica ativação ansiogênica e as consequentes respostas evasivas.

Artigo submetido em 20/07/2020, aceito em 21/07/2020. Os autores informam não haver conflitos de interesse associados à publicação deste artigo.

Fontes de financiamento inexistentes.

Correspondência: Leonardo Marengo, Facultad de Psicología, Universidad Nacional de Córdoba, Bv. de La Reforma esq. Enfermera Gordillo s/n., CP 5000, Córdoba, Argentina. E-mail: Imarengo66@gmail.com

\section{Referências}

1. Marengo L, Ferreira LN, Rivero TS, Godoy JC. A critical view of a treatment intervention for stuttering based on video games. Int J Psychol Neurosci. 2015;2:235-50.

2. Lavender TJ, Gromala D. Audition, the game. Procedia Comput Sci. 2012;15:187-92.

3. American Psychiatric Association. Diagnostic and Statistical Manual of Mental Disorders, Fifth Edition (DSM-5). Arlington: American Psychiatric Publishing; 2013.

4. Perez HR, Stoeckle JH. Stuttering: clinical and research update. Can Fam Physician. 2016;62: 479-84.

5. Walsh B, Mettel KM, Smith A. Speech motor planning and execution deficits in early childhood stuttering. J Neurodev Disord. 2015;7:27.

6. The Stuttering Foundation. Electronic devices, software and Apps [Internet]. 2019 [cited 2020 Oct 13]. www.stutteringhelp.org/electronicdevices-software-and-apps 
7. Fernández-Zúñiga $A$, de León $M$. Conceptos básicos y abordaje de la tartamudez. FMC. 2011:18:193-9.

8. Reilly S, Onslow M, Packman A, Cini E, Conway L, Ukoumunne OC, et al. Natural history of stuttering to 4 years of age: a prospective community-based study. Pediatrics. 2013;132:460-7.

9. McAllister J, Collier J, Shepston L. The impact of adolescent stuttering and other speech problems on psychological well-being in adulthood: evidence from a birth cohort study. Int J Lang Commun Disord. 2013;48:458-68.

10. Felsenfeld S, Kirk KM, Zhu G, Statham DJ, Neale MC, Martin NG. A study of the genetic and environmental etiology of stuttering in a selected twin sample. Behav Genet. 2000;30:359-66.

11. Craig A, Tran Y. Trait and social anxiety in adults with chronic stuttering: conclusions following meta-analysis. J Fluency Disord. 2014;40:35-43.

12. Helgadottir FD, Menzies RG, Onslow M, Packman A, O'Brian S. A standalone Internet cognitive behavior therapy treatment for social anxiety in adults who stutter: CBTpsych. J Fluency Disord. 2014;41:47-54.

13. Brignell A, Krahe $M$, Downes $M$, Kefalianos E, Reilly S, Morgan AT. A systematic review of interventions for adults who stutter. J Fluency Disord. 2020;64:105766.

14. Mongia M, Gupta AK, Vijay A, Sadhu R. Management of stuttering using cognitive behavior therapy and mindfulness meditation. Ind Psychiatry J. 2019;28:4-12.

15. Boyle MP. The impact of causal attribution on stigmatizing attitudes toward a person who stutters. J Commun Disord. 2016;60:14-26.

16. Rodríguez-Carvajal R, Moreno-Jiménez JE, García-Rubio C, Castillo R, Montero I. Efectos del entrenamiento breve en mindfulness en tartamudez: un estudio de caso único [Intetrnet]. 2017 [cited 2020 Oct 13]. blogs.ua.es/aesppu/ files/2017/10/13Me14-Tartamudez.pdf

17. Harley J. The Role of attention in therapy for children and adolescents who stutter: cognitive behavioral therapy and mindfulness- based interventions. Am J Speech Lang Pathol. 2018;27:1139-51.

18. Beilby JM, Byrnes ML, Yaruss JS. Acceptance and commitment therapy for adults who stutter: psychosocial adjustment and speech fluency. J Fluency Disord. 2012;37:289-99.

19. De Veer S, Brouwers A, Evers W, Tomic W. A pilot study of the psychological impact of the mindfulness-based stress reduction program on persons who stutter. Eur J Psychother Couns. 2009;9:39-56.

20. Ingham RJ, Ingham JC, Bothe AK, Wang Y, Kilgo M. Efficacy of the modifying phonation intervals (MPI) stuttering treatment program with adults who stutter. Am J Speech Lang Pathol. 2015;24:256-71.

21. Chesters J, Möttönen R, Watkins KE. Transcranial direct current stimulation over left inferior frontal cortex improves speech fluency in adults who stutter. Brain. 2018;141:1161-71.

22. Rivero T. A construção de um método para o desenvolvimento de um videogame para treino de controle inibitório para adolescentes com transtorno do déficit de atenção e hiperatividade [dissertation]. São Paulo: Universidade Federal de São Paulo; 2016.

23. Baranowski T, Buday R, Thompson D, Lyons EJ, Lu AS, Baranowski J. Developing games for health behavior change: getting started. Games Health J. 2013;2:183-90.

24. Rivero TS, Núñez LM, Pires EU, Bueno OF. ADHD rehabilitation through video gaming: a systematic review using PRISMA guidelines of the current findings and the associated risk of bias. Front Psychiatry. 2015;6:151.

25. Scheurich JA, Beidel, DC, Vanryckeghem $M$. Exposure therapy for social anxiety disorder in people who stutter: an exploratory multiple baseline design. J Fluency Disord. 2019;59:21-32.

26. Klinger E, Bouchard S, Légeron P, Roy S, Lauer F, Chemin I, et al. Virtual reality therapy versus cognitive behavior therapy for social phobia: a preliminary controlled study. Cyberpsychol Behav. 2005;8:76-88. 